\title{
BMJ Open Rationale and protocol for the efficacy, safety and tolerability of nangibotide in patients with septic shock (ASTONISH) phase IIb randomised controlled trial
}

\author{
Bruno Francois, ${ }^{1}$ Simon Lambden (1) , ${ }^{2}$ Sebastien Gibot, ${ }^{3}$ Marc Derive, ${ }^{4}$ \\ Aurelie Olivier, ${ }^{4}$ Valerie Cuvier, ${ }^{4}$ Stephan Witte, ${ }^{4}$ Jean-Marie Grouin, ${ }^{5}$ \\ Jean Jacques Garaud, ${ }^{4}$ Margarita Salcedo-Magguilli, ${ }^{4}$ Mitchell Levy, ${ }^{6}$ \\ Pierre-François Laterre ${ }^{7}$
}

To cite: Francois B, Lambden S, Gibot S, et al. Rationale and protocol for the efficacy, safety and tolerability of nangibotide in patients with septic shock (ASTONISH) phase $\mathrm{llb}$ randomised controlled trial. BMJ Open 2021;11:e042921. doi:10.1136/ bmjopen-2020-042921

- Prepublication history and additional online supplemental material for this paper are available online. To view these files, please visit the journal online. To view these files, please visit the journal online (http://dx.doi.org/10.1136/ bmjopen-2020-042921).

Received 19 July 2020 Accepted 01 June 2021

Check for updates

(c) Author(s) (or their employer(s)) 2021. Re-use permitted under CC BY-NC. No commercial re-use. See rights and permissions. Published by BMJ.

For numbered affiliations see end of article.

Correspondence to Dr Simon Lambden; spl48@cam.ac.uk

\section{ABSTRACT}

Introduction Septic shock is the subgroup of patients with sepsis, which presents as vasopressor dependence, an elevated blood lactate concentration and is associated with a mortality of at least $30 \%$. Expression of the triggering receptor expressed on myeloid cells 1 (TREM-1) pathway, measured using a serum biomarker of pathway activation (soluble TREM-1, sTREM-1) has been associated with outcome in septic shock. Preclinical and early phase patient data suggest that therapeutic modulation of this pathway may improve survival.

Methods and analysis Efficacy, Safety and Tolerability of Nangibotide in Patients with Septic Shock is a phase llb randomised controlled trial that will take place in up to 50 centres in seven countries and recruit 450 patients with septic shock to receive either placebo or one of two doses of nangibotide, a novel regulator of the TREM-1 pathway. The primary outcome will be the impact of nangibotide therapy on the change in Sequential Organ Failure Assessment score from a baseline determined before initiation of study drug therapy. This will be assessed first in the patients with an elevated sTREM-1 level and then in the study population as a whole. In addition to safety, secondary outcomes of the study will include efficacy of nangibotide in relation to STREM-1 levels in terms of organ function, mortality and long-term morbidity. This study will also facilitate the development of a novel platform for the measurement of sTREM-1 at the point of care.

Ethics and dissemination The study has been approved by the responsible ethics committees/institutional review boards in all study countries: Belgium: Universitair Ziekenhuis Antwerpen, France: CPP Ile de France II, Denmark: Region Hovedstaden, Spain: ethics committee from Valld'Hebron Hospital, Barcelona, Finland: Tukija, Ireland: St. James' Hospital (SJH) / Tallaght University Hospital (TUH) Joint Research Ethics Committee, USA: Lifespan, Providence Trial registration numbers EudraCT Number: 2018004827-36 and NCT04055909.

\section{INTRODUCTION}

Sepsis is defined as a life-threatening condition arising as a result of the body's dysregulated host response to infection. ${ }^{1}$ Septic

\section{Strengths and limitations of the study}

- The multicentre Efficacy, Safety and Tolerability of Nangibotide in Patients with Septic Shock study will address the efficacy of a novel therapeutic agent nangibotide at two doses in improving acute morbidity and mortality of patients with septic shock in up to 50 hospitals in at least six countries.

- The primary endpoint, the change in Sequential Organ Failure Assessment score from baseline to day 5 will offer insights into the impact of treatment on acute morbidity and provide a robust surrogate to facilitate design of future trials.

- The primary outcome will be assessed in patients with an elevated soluble triggering receptor expressed on myeloid cells 1 level (a marker of pathway activation) and in the overall population

- Secondary outcomes will assess the impact of nangibotide therapy on mortality and morbidity in septic shock.

- As a phase Ilb trial, the study is not powered to detect an impact on mortality in septic shock and utilises a validated surrogate to detect clinically relevant benefit.

shock is present in the subgroup of patients with sepsis, persistent hypotension requiring vasopressor support and an elevated serum lactate. ${ }^{2}$ This multisystem disorder is associated with a $30 \%$ mortality and substantial morbidity including a higher risk of mortality during succeeding years, ${ }^{34}$ as well as cognitive and physical complications, immune dysfunction, secondary infections, persistent organ damage, impaired quality of life and depression or post-traumatic stress. ${ }^{56}$

The current recommendations for treatment of septic shock remain largely supportive $^{7}$ in spite of extensive efforts to develop new therapies. ${ }^{8}$ Novel therapeutic approaches that have shown promise in 
preclinical development have repeatedly failed in clinical trial. ${ }^{10}$ This has led to recognition that conventional randomised controlled trial designs in sepsis may be inadequate for the development of new therapies and that new trial designs that target specific populations within the septic shock population are required. ${ }^{911}$

The triggering receptor expressed on myeloid cells 1 (TREM-1) is an immunomodulatory receptor expressed on innate immune cells, endothelial cells and platelets. ${ }^{12-15}$ The biological function of TREM-1 is the amplification of the inflammatory response. In sepsis, this may contribute to the dysregulated immune response,${ }^{16}$ which plays a role in the development and progression of septic shock. Exaggerated activation of this pathway, measured by high circulating levels of expression of the cleaved portion of the receptor soluble TREM-1 (sTREM-1), is associated with increased mortality in patients with septic shock. $^{17}$

Nangibotide is a 12 amino-acid peptidic fragment derived from TREM-like transcript-1, a receptor protein belonging to the TREM-1 family. Nangibotide can bind the TREM-1 ligand and thereby modulate the amplification of the immune response caused by the activation of the TREM-1 pathway in sepsis. ${ }^{18} 19$

Extensive preclinical modelling in rodent, porcine and primate septic shock revealed a protective effect of nangibotide in terms of organ function, cardiovascular status and survival. ${ }^{20} 21$ A recent phase IIa clinical trial investigated the safety and tolerability of three doses of nangibotide for up to 5 days in 49 patients with septic shock. In this trial, treatment with nangibotide was found to be safe and well tolerated. Although this study was not designed to prove efficacy, nangibotide treated patients demonstrated numerical improvements in markers of organ function consistent with the hypothesis that TREM-1 inhibition may improve outcomes in septic shock patients. This effect was larger in the subgroup of patients with high circulating levels of sTREM-1. ${ }^{22}$

We report the clinical and statistical design of the Efficacy, Safety and Tolerability of Nangibotide in Patients with Septic Shock (ASTONISH), a randomised, doubleblind, placebo-controlled dose selection phase IIb study in patients with septic shock. The study includes a number of innovative components to address the limitations of previous studies in this area and a novel analytical approach to determining the primary outcome.

\section{METHODS AND ANALYSIS Objectives \\ Primary}

- To evaluate the efficacy of two doses of nangibotide on organ dysfunction (Sequential Organ Failure Assessment, SOFA score) in patients with septic shock in relation to their sTREM-1 plasma levels (patients with high sTREM-1 levels at baseline and all patients).

\section{Secondary}

Secondary objectives of the study are to investigate the safety and efficacy of two doses of nangibotide in patients with septic shock in relation to their sTREM-1 levels.

This includes:

- To evaluate the effect of nangibotide on mortality for up to 12 months.

- To evaluate the effect of nangibotide on other clinical parameters (eg, duration of shock, vasopressor use, ventilator and renal replacement use, secondary infections).

- To evaluate the safety and tolerability of nangibotide.

- To evaluate the effect of nangibotide on quality of life, resource utilisation and postshock morbidity.

Box 1 Inclusion and exclusion criteria for the Efficacy, Safety and Tolerability of Nangibotide in Patients with Septic Shock study

\section{Inclusion criteria}

- Provide written informed consent (proxy/legal representative) according to local regulations.

- Age 18-85 years (inclusive).

- Documented or suspected infection: lung, abdominalor, in patients aged $\geq 65$ years, urinary tract infection.

- Organ dysfunction defined as acute change in total Sequential Organ Failure Assessment score $\geq 2$ points.

- Refractory hypotension requiring vasopressors to maintain MAP $\geq 65 \mathrm{~mm} \mathrm{Hg}$ despite adequate volume resuscitation (as per recommendations of the Surviving Sepsis Campaign).

- Hyperlactataemia (blood lactate $>2 \mathrm{mmol} / \mathrm{L}$ or $18 \mathrm{mg} / \mathrm{dL}$ ). This criterion must be met at least once for the purpose of diagnosis within the 24 hours before study drug administration.

\section{Exclusion criteria}

- Previous episode of septic shock requiring vasopressor administration within current hospital stay.

- Underlying concurrent immunodepression with anti-CD52 alemtuzumab or glucocorticoids $>75 \mathrm{mg}$ prednisone daily or equivalent for more than 7 days days.

- Immunosuppressive therapy related to recent (<6 months) transplantation.

- Cancer chemotherapy ( $<3$ months) implying an immunodepression.

- Known HIV infection with low CD4 cell count $(<200)$ for at least 6 months.

- Known pregnancy (positive urine or serum pregnancy test).

- Shock of any other cause.

- Ongoing documented or suspected endocarditis, history of prosthetic heart valves.

- Prolonged QT syndrome.

- End-stage neurological disease.

- End-stage cirrhosis (Child Pugh Class C).

- Acute Physiology and Chronic Health Evaluation score $<15$ or $\geq 34$.

- Home oxygen therapy on a regular basis for $>6$ hours/day.

- Recent cardiopulmonary resuscitation (within current hospital stay).

- Body mass index $\geq 40 \mathrm{~kg} / \mathrm{m}^{2}$ or weight $\geq 130 \mathrm{~kg}$.

- Moribund patients.

- Decision to limit full care taken before obtaining informed consent.

- Participation in another interventional study in the 3 months prior to randomisation. 


\section{Exploratory}

- To evaluate PK/PD relationship to nangibotidemechanism-of-action-related markers.

\section{ASTONISH trial design}

This is a multicentre randomised, double-blind, placebocontrolled dose-selection study in which two doses of nangibotide will be tested versus placebo. It will take place in approximately 50 centres in seven countries: France, Belgium, Denmark, Finland, Spain, Ireland and the USA. The study was initiated in November 2019. Additional sites may be added depending on recruitment rate.

\section{Eligibility}

All patients with a diagnosis of septic shock will be considered for study participation. The applicable local requirements for informed consent will be followed. All patients will receive standard of care for the treatment of septic shock.

After screening for eligibility by a central coordinating centre, patients meeting the inclusion and exclusion criterion will be consented by the site team and randomised (box 1).

\section{Study drug}

All patients will be treated with standard therapy for septic shock. In addition, patients will receive a loading dose of nangibotide over fifteen minutes followed by infusion at one of two doses $(6.66 \mathrm{mg} / \mathrm{kg}+0.3 \mathrm{mg} / \mathrm{kg} /$ hour or $20 \mathrm{mg} / \mathrm{kg}+1.0 \mathrm{mg} / \mathrm{kg} /$ hour) or a matched placebo.

Treatment with study drug must be initiated as early as possible, but no later than 24 hours after the onset of septic shock, defined by the start of vasopressor therapy. Blood samples for pharmacokinetic and exploratory pharmacodynamic analyses will be collected before, during and after the treatment period.

Patients will be treated for at least 3 days ( $72 \pm 2$ hours) with study drug or until $24( \pm 2)$ hours after vasopressor withdrawal with a maximum treatment duration of 5 days

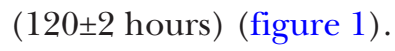

\section{Blinding}

ASTONISH is a double-blind trial. Study personnel including investigators, patients, sponsor and contracted research organisations will be blinded to treatment allocation until closure of the primary endpoint data set at day
28. Unblinding will only take place if knowledge of the patient treatment allocation would facilitate emergency treatment. The investigational drug and the placebo are indistinguishable and presented in the same way.

\section{Endpoints}

Primary endpoint:

The primary endpoint is the change of total SOFA score from baseline to day 5 , which will be assessed in the subgroup defined by patients with elevated sTREM-1 baseline levels $(\geq 400 \mathrm{pg} / \mathrm{mL})$ and in the overall population.

Prespecified secondary endpoints will include:

Efficacy parameters:

Key secondary endpoint.

- All-cause mortality on day 28.

Secondary endpoints.

- Duration of Intensive Care Unit (ICU) stay, hospitalisation.

- Organ support-free survival.

- Daily change of total SOFA score and subscores

- Time until shock reversal defined as cessation of vasopressor support for 24 hours

- Vasopressor use.

- Invasive mechanical ventilation.

- Renal support.

- Overall survival at 90 days, 6 and 12 months.

- Septic shock-related mortality up to 90 days, 6 and 12 months.

- Incidence of secondary infections and postshock antibiotic use.

Safety parameters:

- Vital signs.

- ECG.

- Safety laboratory tests: haematology, coagulation, plasma biochemistry.

- Presence of antinangibotide antibodies.

- AEs, SAEs and deaths.

Pharmacokinetics:

Nangibotide plasma levels

Pharmacodynamics (exploratory): sTREM-1, immune and vascular-related biomarkers.

Pharmacoeconomic endpoints up to 12 months:

- Health-related quality of life (EQ-5D).

- Postshock morbidity.

- Healthcare resource utilisation.

Figure 1 Study flow chart. CCC, Central Co-ordinating centre; EoS, end of study; Fu, follow-up; LD, loading dose or matching placebo. 
Patients will be assessed at the End of Study visit at day 28. After the last patient's day 28 visit, the study data will be analysed. Additional follow-up (FU) visits will be conducted after 90 days, 6 and 12 months. Quality of life, morbidity and survival status of patients will be collected at these visits which may be undertaken remotely in order to minimise loss of patients to FU. Data from the long-term FU will be analysed and reported separately (a complete list of study investigations and their timing is presented in online supplemental files 1 and 2). Adverse event (AE) data will be collected until day 28, drug-related serious AEs (SAEs) can be reported without time limit.

sTREM-1 is a mechanism-based plasma marker associated with activation of the TREM-1 signalling pathway. As such it may be a biomarker predictive of treatment response to nangibotide. The plasma levels of sTREM-1 will be determined at the second interim analysis and at the end of the study for the biostatistical analysis.

\section{Randomisation and sample size}

Following screening for eligibility, study centres will contact a central, independent coordinating centre to confirm eligibility. They will then be issued with a unique randomisation code that will facilitate identification of the correct blinded allocation of study drug.

The randomisation will be stratified according to site and patients will be allocated on a 1:1:1 basis to one of three treatment arms. The randomisation scheme will be generated by an independent statistician who is not part of the study team. A randomisation number will be assigned to each patient. The randomisation assignment will be implemented by an interactive response technology.

Results $^{23}$ from a previous pilot phase II study (MOT-C201) have shown that a difference of around two versus placebo in mean changes of the primary endpoint (accounting for missing values occurring prior to day 5 ) and an SD of around 3.3, could be expected in the overall population, and even higher than two in the subgroup of patients with elevated sTREM-1 levels. It is expected that the high sTREM-1 subgroup will comprise at least half the patients enrolled (around 225 patients) in the study.

Considering 450 patients (150 patients per treatment arm), this study has at least $90 \%$ power to detect the expected difference in at least one dose either in the subgroup of patients with elevated sTREM-1 levels or in the overall population.

\section{Analysis plan}

This exploratory study has three parallel aims

- Assessment of the safety and tolerability of two doses of nangibotide.

- Assessment of the efficacy of two doses of nangibotide on the primary and secondary efficacy endpoints.

- Determining the best cut-off for baseline sTREM1 as a predictive biomarker.

The primary efficacy variable is the change of SOFA from baseline to day 5 . This will be tested in the subgroup defined by patients with elevated sTREM-1 baseline levels and in the overall population. This will be based on an analysis of covariance model adjusting for randomised treatment and the baseline SOFA score.

The primary analysis will be undertaken in the modified intention to treat set (all randomised patients having received at least one dose of the prescription of study drug (either nangibotide or placebo).

Missing values occurring prior to day 5 are likely to be missing not at random and will be replaced as follows:

- Missing SOFA values not due to death will be replaced by the last available postrandomisation value of the SOFA score (ie, last observation carried forward method).

- Missing values due to death will be replaced by the last available postrandomisation value of the total SOFA score increased by an additional penalty of four points.

Sensitivity analyses using different penalty scores and other methods for handling missing data will be performed and described in the Statistical Analysis Plan (SAP).

This is a phase IIb trial, therefore, no adjustment for multiplicity will be proposed for the primary analysis and tests for nangibotide doses versus placebo comparisons will be performed at the usual nominal one-sided alpha level of 0.025 both in the subgroup and the overall population. However, a secondary adjusted analysis will be performed to account for multiplicity and control the overall one-sided error rate to 0.025 : details will be further provided in the SAP before the unblinding of the database.

The key secondary endpoint of 28-day mortality will be assessed using a log-rank test to compare the treatment arms. In addition, a proportional hazard Cox model adjusting for the same covariates used for the primary endpoint analysis will be fitted to estimate the treatment effect.

Details of other endpoint analyses are described in the SAP.

The threshold for categorising high and low sTREM-1 is defined as $400 \mathrm{pg} / \mathrm{mL}$ at baseline and is based on analysis of biomarker and outcome data from the phase IIa study. ${ }^{23}$ However, exploratory analyses based on the current study will be conducted to confirm the optimal cut-off for future studies.

After the first 113 (around 25\%) and 225 (50\%) patients have completed the 7-day period after randomisation, unblinded interim analyses of safety will be performed and the data reviewed by an independent data monitoring committee (DMC). The second interim analysis will, in addition, include an analysis of efficacy data, after which the DMC will make recommendations for stopping the clinical trial or a treatment arm for futility (see online supplemental file 3 for DMC Charter). As well, if the proportion of patients with high sTREM-1 baseline values is less than $50 \%$ of the total, then the planned total sample size may be increased to maintain the power of the subgroup analyses. Data will be validated using on 
site visits and source data verification by blinded clinical research associates.

\section{PATIENT AND PUBLIC INVOLVEMENT}

No patient involved.

\section{Ethics and dissemination}

Ethical approval has been secured in Belgium, France, Denmark, Finland, Ireland and Spain in Europe and in the USA.

Consent for this study cannot be secured from a substantial proportion of eligible study participants due to lack of capacity associated with their diagnosis of septic shock. An emergency informed consent procedure will be applied according to applicable regulations and to the approval of the respective ethics committe or institutional review board in patients who are assessed by their treating clinician as lacking capacity. Consent for further data/sample collection may be withdrawn by the patient at any time after they have regained capacity (an example consent form is provided in online supplemental file 4).

A manuscript with the results of the primary study will be published in a peer-reviewed journal and anonymised throughout. Separate manuscripts may be written on the secondary aims, and these will also be submitted for publication in peer-reviewed journals. Results will also be disseminated at national and international meetings (see online supplemental file 5) for Standard Protocol Items: Recommendations for Interventional Trials checklist).

\section{DISCUSSION}

This study has several novel components in terms of study design and analysis.

Nangibotide is the first compound to reach clinical trial that targets the critical TREM-1 pathway. This study will explore the efficacy of nangibotide in septic shock patients with elevated baseline levels of sTREM-1, a biomarker that reflects excessive activation of the target pathway and is associated with increased mortality. This approach may provide evidence for the design of a future phase III trial utilising a personalised, mechanism based biomarker to select patients likely to benefit from nangibotide.

In addition to selecting patients based on the clinical presentation of septic shock, this study employs additional prognostic enrichment by excluding those patients with Acute Physiology and Chronic Health Evaluation scores less than 15 or greater than or equal to 34 at baseline. This approach excludes patients at a low risk of death and those with a high estimated mortality $\left(\geq 85 \%{ }^{24}\right.$ for whom nangibotide treatment is unlikely to be of benefit, reduces the outcome heterogeneity within the septic shock population and increases the likelihood of detecting a clinically relevant benefit.

The statistical analysis plan for this study includes the analysis of the primary endpoint in both the whole study population and the subgroup of patients with high sTREM-1 levels. This proposed stepwise analytical approach will offer adequate power to detect the efficacy of nangibotide in both patient groups while preserving confidence that a false positive result is unlikely .

The challenge of detecting clinically relevant benefit in phase II studies of novel therapeutic agents in septic shock is a substantial one which has been recognised by the clinical community ${ }^{25}$ and regulators. ${ }^{26}$ Change in SOFA, an established marker of the acute morbidity associated with sepsis, has been consistently shown to act as a surrogate for subsequent mortality in septic shock. ${ }^{27} 28$ By incorporating robust guidelines for collection of clinical information and the handling of missing data, ${ }^{28}$ this study may improve the consistency and handling of data of this kind in randomised controlled trials.

Limitations of this phase IIb study include the use of a surrogate endpoint to detect clinically relevant efficacy. While the change in SOFA score has been extensively validated, further studies will be required to demonstrate improved mortality as a primary outcome. Furthermore, by defining a priori the subgroup of patients with elevated sTREM-1 levels as those most likely to benefit, the available statistical power to detect a benefit only in those patients with low sTREM-1 levels at baseline is reduced.

Specific treatments for septic shock have remained elusive and given the increasing prevalence, substantial mortality and morbidity that is associated with it, the demand for novel therapies to treat the condition continues to be high. With increasing recognition that a variety of endotypes exist within the population characterised by the standard definitions, ${ }^{29}{ }^{30}$ novel agents targeting subgroups most likely to benefit from treatment offer an attractive approach. Nangibotide is the first agent to target the TREM-1 pathway in patients, and the ASTONISH trial will provide valuable insights into both the safety and efficacy of this novel therapy in the treatment of patients with septic shock.

\section{Author affiliations}

${ }^{1}$ Medical-Surgical ICU Department and Inserm CIC1435 \& UMR1092, CRICS-TRIGGERSEP Network, University of Limoges, Limoges, France

${ }^{2}$ Department of Medicine, University of Cambridge, Cambridge, UK

${ }^{3}$ Department of Intensive care medicine, CHRU de Nancy, Nancy, France

${ }^{4}$ Inotrem SA, Paris, France

${ }^{5}$ Universite de Rouen, Mont-Saint-Aignan, France

${ }^{6}$ Division of Pulmonary, Critical Care and Sleep Medicine, Department of Medicine, Brown University School of Medicine, Providence, Rhode Island, USA

${ }^{7}$ Department of Critical Care Medicine, Cliniques Universitaires Saint-Luc, Brussels, Belgium

\section{Twitter Simon Lambden @simonlambden}

Contributors SL drafted the manuscript. SL, SW, MD, SG, A0, VC, BF, P-FL, ML, JJG, MS-M and J-MG all contributed to the development and design of the protocol, reviewed the manuscript and approved the final version.

Funding This work is supported by Inotrem SA, no grant number is available.

Competing interests The authors have the following interests: SL, MS-M, JJG, SW and VC are employees of Inotrem SA. BF is the principal investigator of the trial and is a member of the steering committee.

Patient consent for publication Not required.

Provenance and peer review Not commissioned; externally peer reviewed. 
Supplemental material This content has been supplied by the author(s). It has not been vetted by BMJ Publishing Group Limited (BMJ) and may not have been peer-reviewed. Any opinions or recommendations discussed are solely those of the author(s) and are not endorsed by BMJ. BMJ disclaims all liability and responsibility arising from any reliance placed on the content. Where the content includes any translated material, BMJ does not warrant the accuracy and reliability of the translations (including but not limited to local regulations, clinical guidelines, terminology, drug names and drug dosages), and is not responsible for any error and/or omissions arising from translation and adaptation or otherwise.

Open access This is an open access article distributed in accordance with the Creative Commons Attribution Non Commercial (CC BY-NC 4.0) license, which permits others to distribute, remix, adapt, build upon this work non-commercially, and license their derivative works on different terms, provided the original work is properly cited, appropriate credit is given, any changes made indicated, and the use is non-commercial. See: http://creativecommons.org/licenses/by-nc/4.0/.

\section{ORCID iD}

Simon Lambden http://orcid.org/0000-0003-4531-4524

\section{REFERENCES}

1 Singer M, Deutschman CS, Seymour CW, et al. The third International consensus definitions for sepsis and septic shock (sepsis-3). JAMA 2016;315:801-10.

2 Shankar-Hari M, Phillips GS, Levy ML, et al. Developing a new definition and assessing new clinical criteria for septic shock: for the third International consensus definitions for sepsis and septic shock (sepsis-3). JAMA 2016;315:775-87.

3 Lone NI, Walsh TS. Impact of intensive care unit organ failures on mortality during the five years after a critical illness. Am J Respir Crit Care Med 2012;186:640-7.

4 Lone NI, Gillies MA, Haddow C, et al. Five-Year mortality and hospital costs associated with surviving intensive care. Am J Respir Crit Care Med 2016;194:198-208.

5 Chang HJ, Lynm C, Glass RM. JAMA patient page. sepsis. JAMA 2010;304:1856.

6 Iwashyna TJ, Ely EW, Smith DM, et al. Long-Term cognitive impairment and functional disability among survivors of severe sepsis. JAMA 2010;304:1787-94.

7 Levy MM, Evans LE, Rhodes A. The surviving sepsis campaign bundle: 2018 update. Intensive Care Med 2018;44:925-8.

8 Opal SM, Dellinger RP, Vincent JL. The next generation of sepsis clinical trial designs: what is next after the demise of recombinant human activated protein C? Crit Care Med 2014 (published Online First: 2014/04/11).

9 Angus DC. The search for effective therapy for sepsis: back to the drawing board? JAMA 2011;306:2614-5.

10 Lambden S, Summers C. Bridging the translational gap: the challenges of novel drug development in critical care. In: Vincent J-L, ed. Annual update in intensive care and emergency medicine 2017. Cham: Springer International Publishing, 2017: 375-88.

11 Cohen J, Vincent J-L, Adhikari NKJ, et al. Sepsis: a roadmap for future research. Lancet Infect Dis 2015;15:581-614.
12 Bouchon A, Dietrich J, Colonna M. Cutting edge: inflammatory responses can be triggered by TREM-1, a novel receptor expressed on neutrophils and monocytes. J Immunol 2000;164:4991-5.

13 Bouchon A, Facchetti F, Weigand MA, et al. Trem-1 amplifies inflammation and is a crucial mediator of septic shock. Nature 2001;410:1103-7.

14 Derive M, Massin F, Gibot S. Triggering receptor expressed on myeloid cells -1 as a new therapeutic target during inflammatory diseases. Self Nonself 2010;1:225-30.

15 Washington AV, Schubert RL, Quigley L, et al. A TREM family member, TLT- 1 , is found exclusively in the $\alpha$-granules of megakaryocytes and platelets. Blood 2004;104:1042-7.

16 Xiao W, Mindrinos MN, Seok J, et al. A genomic storm in critically injured humans. J Exp Med 2011;208:2581-90.

17 Brenner T, Uhle F, Fleming T, et al. Soluble TREM-1 as a diagnostic and prognostic biomarker in patients with septic shock: an observational clinical study. Biomarkers 2017;22:63-9.

18 Washington AV, Gibot S, Acevedo I, et al. TREM-like transcript-1 protects against inflammation-associated hemorrhage by facilitating platelet aggregation in mice and humans. J Clin Invest 2009;119:1489-501.

19 Derive M, Bouazza Y, Sennoun N, et al. Soluble TREM-like transcript-1 regulates leukocyte activation and controls microbial sepsis. J Immunol 2012;188:5585-92.

20 Lemarié J, Boufenzer A, Popovic B, et al. Pharmacological inhibition of the triggering receptor expressed on myeloid cells- 1 limits reperfusion injury in a porcine model of myocardial infarction. ESC Heart Failure 2015;2:90-9.

21 Derive M, Boufenzer A, Bouazza Y, et al. Effects of a TREM-Like transcript 1-Derived peptide during hypodynamic septic shock in pigs. Shock 2013;39:176-82.

22 François BWX, Ferrer R, Mira JP. Sepsis 2018. Intensive Care Medicine Experimental 2018;6:1-33.

23 François B, Wittebole X, Ferrer R, et al. Nangibotide in patients with septic shock: a phase $2 \mathrm{~A}$ randomized controlled clinical trial. Intensive Care Med 2020;46:1425-37.

24 Knaus WA, Draper EA, Wagner DP, et al. APACHE II: a severity of disease classification system. Crit Care Med 1985;13:818-29.

25 Stanski NL, Wong HR. Prognostic and predictive enrichment in sepsis. Nat Rev Nephrol 2020;16:20-31.

26 European Medicines Agency. CPMP/EWP/4713/2003. guideline on clinical investigation of medicinal products for the treatment of sepsis. Domenico Scarlattilaan, 61083 HS Amsterdam, The Netherlands: European Medicines Agency, 2006.

27 de Grooth H-J, Geenen IL, Girbes AR, et al. SOFA and mortality endpoints in randomized controlled trials: a systematic review and meta-regression analysis. Crit Care 2017;21:38.

28 Lambden S, Laterre PF, Levy MM, et al. The SOFA scoredevelopment, utility and challenges of accurate assessment in clinical trials. Crit Care 2019;23:374.

29 Seymour CW, Kennedy JN, Wang S, et al. Derivation, validation, and potential treatment implications of novel clinical phenotypes for sepsis. JAMA 2019;321:2003.

30 Davenport EE, Burnham KL, Radhakrishnan J, et al. Genomic landscape of the individual host response and outcomes in sepsis: a prospective cohort study. Lancet Respir Med 2016;4:259-71. 